\title{
Collision Detection and Prevention Using the Leksell Gamma Knife
}

\author{
L. Dade Lunsford \\ Department of Neurological Surgery, School of Medicine, The University of Pittsburgh, Pittsburgh, PA, USA
}

As a human interest event for users of the Perfexion or ICON gamma knife, the case presented discloses a rare reason for unexpected stoppage in delivery of the radiation in a gamma knife. Anything that falls on the sensor screen of the internal frame cap of the gamma knife is interpreted by the software as a "collision," which is precisely why it was engineered to interrupt treatment, retract the Cobalt sources, and evict the patient from the unit until the issue is resolved. At our center in Pittsburgh (15,400 gamma knife procedures using U, B, C, 4C, Perfexion, and ICON), we have encountered two similar events. The first was exactly as in the report by Tripathi et al. [1]: the patient was emotionally upset and tearful. Each time a tear hit the internal screen (sensor), out she came. We realized after several times that there were tears dripping from the frame. More sedation and reassurance solved the problem. The second event, perhaps more interesting, was similar in the sense that every few minutes the patient was ejected with no rational reason for collision. In fact, we had done a stereotactic biopsy of the lesion having gamma knife about $2 \mathrm{~h}$ earlier. The resident (it's always the resident you know!) did not tie the suture of the scalp tight enough and a drop of CSF continued to run off the head, frame, and hit the sensor. A reinforced suture solved this problem.

\section{KARGER}

() 2019 S. Karger AG, Basel

E-Mail karger@karger.com

www.karger.com/sfn
We have taught over 2,500 people at our gamma knife courses over the past 20 plus years. During each course, we remind students of the intricate design of the gamma knife and provide a vignette of these two cases. As in all surgical procedures, we sometimes have to be investigative sleuths to solve unexpected events.

Collisions between the stereotactic frame, pins, posts, or skull are relatively rare with either Perfexion or ICON. This was part of the redesign process. Frame placement occasionally requires shifting in anticipation of potential collisions. Shifting is almost never required in the leftright axis (X coordinate). But for inferior $(\mathrm{Z})$ targets, the frame base ring should be placed as low as possible. For anterior $(\mathrm{Y})$ targets, the surgeon should shift the frame anteriorly and use short frame posts in the back to reduce collision risks. For posterior targets, even those at the occipital bone can be solved almost always by shifting the frame posteriorly and by a flexion gamma angle of 110 degrees.

\section{Reference}

1 Tripathi M, Kulshresta A, Oinum A, Kumar N, Batish A, Deora H, Agrawaal A: Tears: A bizarre cause of collision in gamma knife radiosurgery. Stereotact Funct Neurosurg. DOI: 10.1159/000495416.

L. Dade Lunsford, MD

Department of Neurological Surgery, School of Medicine, The University of Pittsburgh 200 Lothrop Street, Suite B-400/461

Pittsburgh, PA 15213 (USA)

E-Mail lunsfordld@ upmc.edu 